\title{
Formas clínicas de la tuberculosis infantil: Hospital Roberto del Río. 1989-2005
}

\author{
GLORIA SÁNCHEZ M.*, ROSSANA MAMANI J.**, JAVIERA RETAMAL M.*, \\ ANGÉLICA ROJO L.* y CARLOS CASAR C.*
}

\section{Clinical forms of tuberculosis in a Chilean children's hospital, 1989-2005}

In Chilean children the incidence of tuberculosis (TB) is low: 2.3/100.000 in younger than 15 years old (2005). This fact may explain that TB diagnosis is sometimes difficult to do in pediatric patients. Our aim was to describe the clinical forms of presentation of TB in children's hospital patients with confirmed TB diagnosis between the years 1989-2005. We carried out this retrospective analysis in 49 patients younger than 15 years old (mean: 5.8 years.); 65.5\% of them had a history of contact with an active tuberculosis case. Twenty nine (59.1\%) patients were diagnosed as pulmonary TB, 19 of them (65.5\%) as primary pulmonary TB and the remainder 10 as post-primary pulmonary $T B$. Out of the 20 patients with extrapulmonary $T B$, adenitis was the most frequent $(n=$ $8)$ followed by pleural effusion $(n=7)$. Chest $x$-ray was normal in 10 out of the 49 patients. Major $x$-ray pathological findings were: primary pulmonary TB $38.7 \%(n=19)$, pleural effusion $14.3 \%(n$ $=7)$, and parenchyma air-space consolidation $10.2 \%(n=5)$. We expect this study would contribute to make a much earlier diagnosis and treatment of TB in children.

Key words: Infantile tuberculosis, clinical and radiological features.

\section{Resumen}

En Chile, la incidencia de tuberculosis (TBC) en la edad pediátrica es baja: 2,3/100.000 en menores de 15 años (2005). Este hecho puede explicar que a veces es difícil hacer el diagnóstico de $T B C$ en pacientes pediátricos. Nuestro propósito fue describir las formas de presentación clínica de la TBC en pacientes del hospital de niños Roberto del Río, con diagnóstico confirmado de TBC entre los años 1989 y 2005. Efectuamos un análisis retrospectivo en 49 pacientes menores de 15 años (edad promedio: 5,8 años.); 65,5\% de ellos tenía antecedentes de contacto con TBC activa. A veintinueve pacientes $(59,1 \%)$ se les diagnosticó TBC pulmonar, en 19 de ellos $(65,5 \%)$ se diagnosticó complejo primario y en los 10 restantes la forma progresiva de TBC pulmonar. En los 20 pacientes con TBC extrapulmonar, la adenitis fue la forma más frecuente $(n=8)$, seguida por la pleuresía $(n=7)$. La radiografía de tórax fue normal en 10 de los 49 pacientes. Los principales hallazgos radiológicos fueron complejo primario TBC 38,7\% $(n=19)$, derrame pleural 14,3\% $(n=7)$, y condensación pulmonar 10,2\% $(n=5)$. Esperamos que este estudio contribuya a efectuar más precozmente el diagnóstico y tratamiento de la TBC en niños.

Palabras clave: Tuberculosis infantil, características clínicas y radiológicas.

\section{Introducción}

La tuberculosis (TBC) es una enfermedad infecciosa de curso progresivo y tendiente a la cronicidad, producida por especies del género
Mycobacterium, principalmente $M$. tuberculosis. Afecta principalmente al pulmón, aunque puede comprometer cualquier órgano.

De distribución mundial, esta enfermedad, infecta a 16 millones de personas cada año, de las

\footnotetext{
* Unidad de Broncopulmonar, Hospital Roberto del Río.

** Pediatra, Becada Enfermedades Respiratorias Pediátricas. Universidad de Chile. Hospital Roberto del Río, Unidad de Broncopulmonar.
} 
cuales 7 millones desarrollan la enfermedad activa y 2 millones mueren.

En Chile, la morbilidad en todas sus formas ha disminuido desde 88,5/100.000 habitantes (1972) a 15/100.000 habitantes en el año 2005, y el descenso más acentuado se observa en menores de 15 años (de 23,4 a 2,3/100.000 habitantes). Esta reducción se debe a la mantención en el tiempo del programa Nacional de Control de la Tuberculosis: pesquisa y tratamiento de los casos bacilíferos, estudio de contactos y la cobertura de vacuna BCG.

La tuberculosis afecta todas las edades, de los pacientes infectados un $10 \%$ desarrolla la enfermedad en el curso de sus vidas. La edad influye en forma importante en la gravedad del cuadro clínico. En el primer año de vida la enfermedad es muy grave con predominio de las formas diseminadas: tuberculosis miliar y meningitis tuberculosa que son de elevada letalidad. Las condiciones socioeconómicas adversas son factores de riesgo, ya que favorecen el hacinamiento y así la mayor transmisión del bacilo. La desnutrición y las inmunodeficiencias primarias o secundarias, son importantes elementos a considerar al momento de evaluar el riesgo de infección y por lo tanto, de desarrollar la enfermedad.

En los niños la tuberculosis puede tener diversos síntomas y signos dependiendo del grado de compromiso y ubicación de la lesión, pudiendo ser incluso asintomática. La tuberculosis infantil está siempre en relación a la primoinfección, lo que implica a un adulto bacilífero, el que constituye la principal fuente de contagio.

La forma clásica es la localización pulmonar, que en los niños frecuentemente corresponde a la primoinfección (complejo primario). Este suele ser asintomático, constituyendo frecuentemente un hallazgo en el estudio de contactos de pacientes bacilíferos. Cuando existen síntomas, éstos pueden manifestarse como fiebre, astenia, baja de peso, eritema nodoso, queratoconjuntivitis flictenular, además de síntomas respiratorios inespecíficos. El diagnóstico se confirma con la presencia de complejo primario en la radiografía de tórax asociado a PPD positivo o viraje del PPD.

Las formas pulmonares progresivas se observan principalmente en niños menores de 4 años, sin antecedente de vacuna BCG y en aquellos con inmunodeficiencias. Puede haber progresión a partir de una lesión parenquimatosa o ganglionar.

Existe una forma de tuberculosis infantil con características similares a la del adulto, que afecta principalmente a adolescentes, que se manifiesta por tos, expectoración, ocasionalmente hemoptisis; su bacteriología es por lo general positiva, y la radiología muestra infiltrados y cavitaciones.

Las formas extrapulmonares pueden ser diversas, manifestándose como: adenitis, pleuresía, tuberculosis osteoarticular, renal, genitourinaria, gastrointestinal, ocular o cutánea, entre otras.

Las formas diseminadas: diseminación miliar y meningitis tuberculosa, son las más graves, con una elevada mortalidad y secuelas.

Si bien la incidencia de TBC ha disminuido significativamente gracias a las estrategias de manejo aplicadas por el sistema de salud, sigue siendo un problema preocupante debido al gran número de infectados que eventualmente se hacen bacilíferos; pese a ello, existen escasos estudios de tuberculosis infantil en Chile, que aporten una orientación clínica y epidemiológica más allá de los datos obtenidos del Ministerio de Salud. Nuestro objetivo fue describir la distribución de formas clínicas de tuberculosis infantil, notificada entre los años 1989 y 2005, de la población asignada al consultorio de especialidades del hospital Roberto del Río.

\section{Pacientes y Métodos}

Realizamos un estudio descriptivo retrospectivo de donde se rescataron los pacientes notificados con alguna forma clínica de TBC, menores de 15 años, controlados en el consultorio de especialidades del hospital Roberto del Río, entre los años 1989 y 2005.

Consideramos como diagnóstico de TBC los casos confirmados (aquellos con cuadro clínico de TBC y bacteriología positiva, ya sea con baciloscopía o cultivo de expectoración o de contenido gástrico y/o biopsia positivas en casos extrapulmonares) y los casos probables (aquellos sugerentes de TBC, sin confirmación bacteriológica, que cuentan con viraje tuberculínico o exámenes de laboratorio que sugieren el diagnóstico clínico).

Se confeccionó una base de datos en la que se incluyeron las siguientes características poblacionales: edad, sexo, vacuna BCG, existencia de contacto bacilífero, presencia de estado de inmunosupresión, PPD, motivo de consulta, presentación clínica de TBC (diagnóstico de egreso) y mortalidad. Los datos obtenidos se expresaron en valores promedio y porcentajes. 


\section{Resultados}

Se reclutaron 49 pacientes con diagnóstico clínico de TBC desde el año 1989 hasta el año 2005. Dentro de las características poblacionales encontramos un promedio de edad de 5,8 años con una dispersión de 0 a 14 años 11 meses. La distribución por sexo fue $42,8 \%$ femenino y $57,2 \%$ masculino. Del total de nuestros pacientes, el $77,5 \%(\mathrm{n}=38)$ presentaban cicatriz de vacuna $\mathrm{BCG}$.

Como condición predisponente, sólo un paciente tenía sospecha de inmunosupresión sin diagnóstico específico, siendo éste el único fallecido en nuestro estudio, alcanzando una mortalidad de $2 \%$.

En cuanto a los antecedentes y presentación clínica, 32 pacientes $(65,3 \%)$, tenía antecedente de contacto TBC. Al analizar el diagnóstico clínico, observamos que $29(59,1 \%)$ presentaban TBC pulmonar, de la cual $19(65,5 \%)$ corresponden a complejo primario y 10 casos $(34,5 \%)$ a la forma pulmonar progresiva (Tabla 1). Veinte pacientes $(40,9 \%)$ presentaron una forma extrapulmonar, dentro de ésta, la más frecuente

Tabla 1. Distribución de casos de tuberculosis pulmonar y extrapulmonar

\begin{tabular}{lrlr}
\hline \multicolumn{2}{l}{ TBC pulmonar } & & \multicolumn{2}{l}{ TBC extrapulmonar } \\
\hline Complejo primario & 19 & Adenitis & 8 \\
Progresiva & 10 & Pleuresía & 7 \\
& & Miliar & 2 \\
& & Meningitis & 1 \\
& & Ósea & 1 \\
Total & Cutánea & 1 \\
& $29(59,1 \%)$ & Total & $20(40,9 \%)$ \\
\hline
\end{tabular}

Tabla 2. Distribución de pacientes por diagnóstico radiológico

\begin{tabular}{lr}
\hline Diagnóstico radiológico & n \\
\hline Normal* $^{*}$ & 10 \\
Derrame pleural & 7 \\
Condensación & 5 \\
Complejo primario & 19 \\
Cavitación & 2 \\
Miliar & 2 \\
Otras & 4 \\
\hline
\end{tabular}

* Pacientes con adenitis (9) y forma cutánea (1) fue la adenitis $(\mathrm{n}=8)$ con un $40 \%$, seguida de pleuresía $(n=7)$ con un $35 \%$. El resto de los pacientes corresponden a formas clínicas aisladas como TBC cutánea, ósea, miliar y meningitis, sumando entre todas un $25 \%$ del total de TBC extrapulmonar (Tabla 1).

A todos nuestros pacientes se les realizó radiografía de tórax, que fue normal en 10 casos $(20,4 \%)$. Dentro de los hallazgos patológicos, se encuentra en 7 casos $(14,3 \%)$ la presencia de derrame pleural, en 19 casos $(38,7 \%)$ la imagen de un complejo primario y en $5(10,2 \%)$ una imagen de condensación sin derrame. El $16,4 \%$ restante $(n=8)$ corresponde a otros hallazgos como neumonía cavitada, TBC miliar y atelectasia (Tabla 2).

Se realizó baciloscopía en el $100 \%$ de nuestros pacientes de las cuales resultaron positivas un $14,2 \%(\mathrm{n}=7)$. Existe registro de cultivo realizado en 25 pacientes, el que fue positivo en 7 de ellos (14,2\%). Cabe destacar que en dos pacientes, cuya baciloscopía fue negativa, el cultivo fue positivo, logrando confirmación diagnóstica. En cuanto al PPD se realizó en todos los pacientes siendo reactivo en el $65,3 \%(n=32)$.

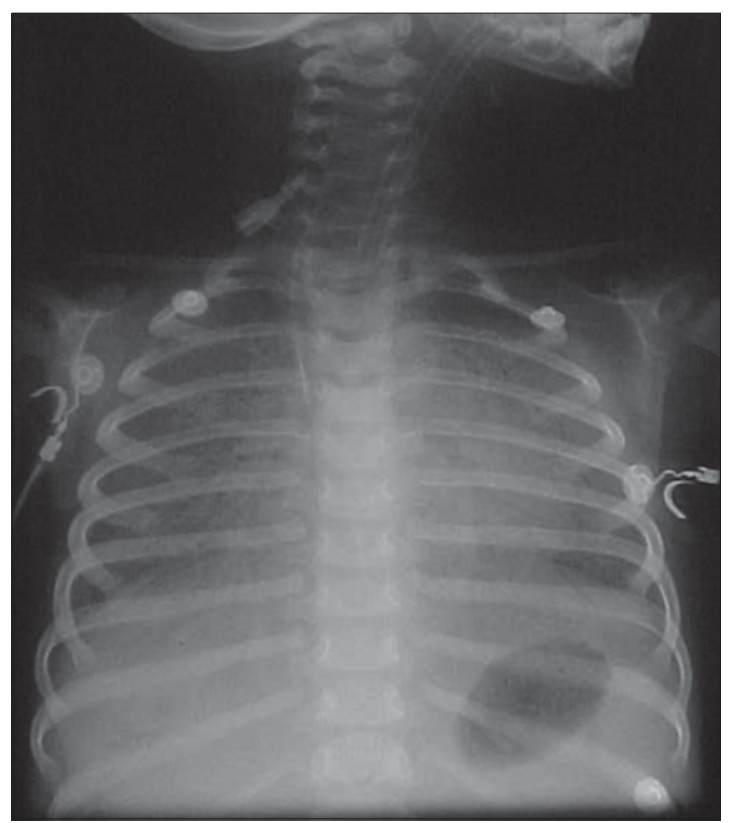

Figura 1. Recién nacido pretérmino 35 semanas; madre con TBC pulmonar bacilífera diagnosticada el día del parto, presenta distress respiratorio severo, requiere VM por 15 días. BK (+) en secreción traqueal, recibe tratamiento, con buena evolución y sin secuelas. La tuberculosis connatal es una forma clínica extremadamente rara. 


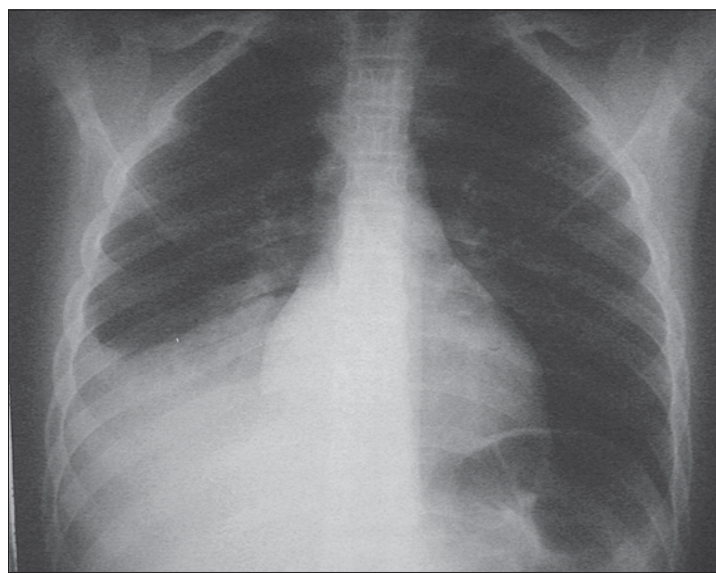

Figura 2. Paciente de 6 años con tos, fiebre y dolor punzante en hemitórax derecho. Líquido pleural citrino, con proteínas elevadas, glucosa $55 \mathrm{mg} / \mathrm{dL}, 1.560$ leucocitos, $85 \%$ mononucleares y adenosin deaminasa en líquido pleural $45 \mathrm{U} / \mathrm{dL}$. Biopsia pleural con aguja muestra tubérculos. Recibe tratamiento completo y continúa sus controles en otra región. Pleuresía serofibrinosa: forma extrapulmonar frecuente.

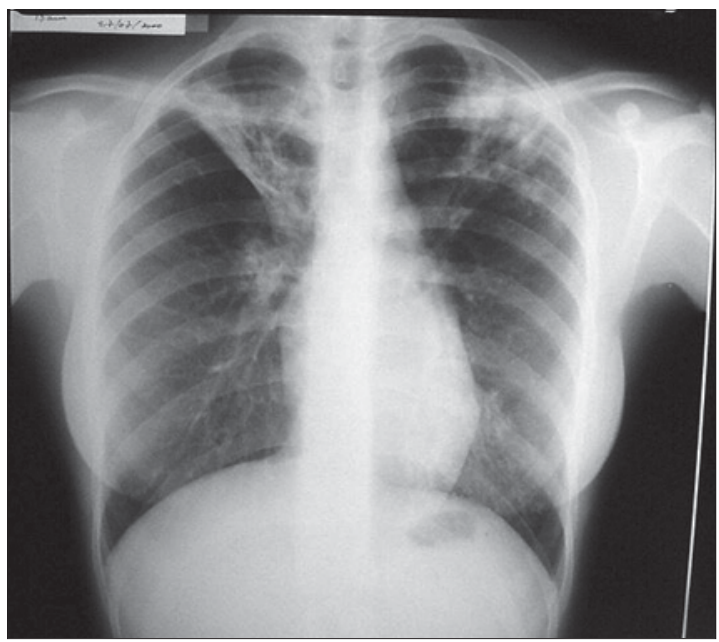

Figura 4. Adolescente, sexo femenino, 13 años, con diagnóstico de neumonía, tratada con macrólidos por 14 días, posteriormente con corticoides inhalatorios durante 10 meses. Persiste con tos y expectoración, agregándose baja de peso, febrículas y crepitaciones en ambos campos pulmonares en el momento de esta radiografía. BK en esputo (+). Recibe tratamiento, quedando con lesiones fibróticas. Tuberculosis tipo adulto.

Todos los pacientes fueron tratados según la Norma Nacional del Programa de Tuberculosis del Ministerio de Salud, Hubo dos abandonos de tratamiento, no hubo recaídas ni reacciones adversas a los medicamentos. El período bajo control fue de 8 a 12 meses.

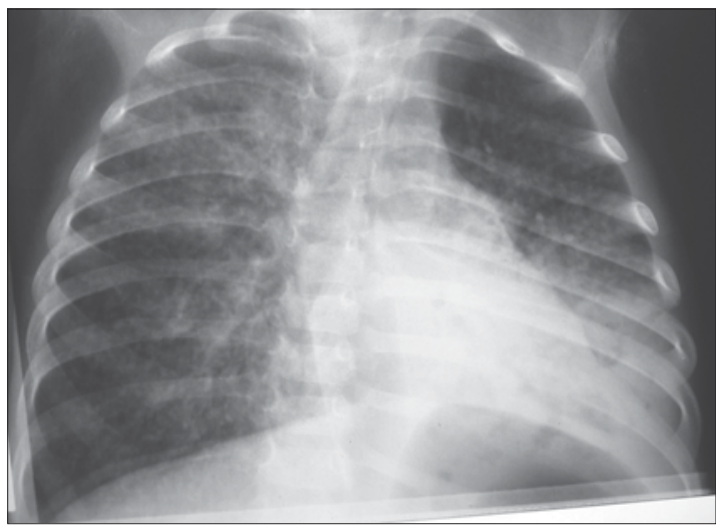

Figura 3. Lactante de 8 meses, procedente de Cochabamba (Bolivia), consulta por cuadro febril prolongado, tos y pobre incremento ponderal. BK en esputo (+). Se diagnostica tuberculosis miliar. No presentó meningitis. Control normal post tratamiento.

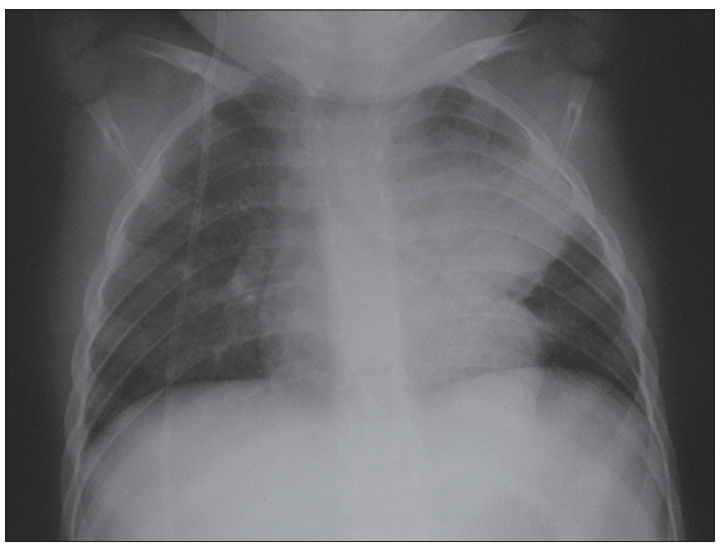

Figura 5. Niña de 3 años 6 meses, portadora de hidrocefalia con válvula derivativa ventrículo-peritoneal. Contacto hermano con TBC pulmonar bacilífera. Escasa tos en últimas 2 semanas. PPD $25 \mathrm{~mm}$. Recibe tratamiento completo según la norma nacional. Asintomática en sus controles con regresión de las imágenes radiológicas. Probable tuberculosis primaria progresiva.

\section{Discusión}

La TBC infantil continúa siendo una patología vigente y de suma importancia debido a la gravedad que puede llegar a alcanzar y a las consecuencias o secuelas que puede provocar el no otorgar un tratamiento oportuno. En los últimos años, Chile ha experimentado un descenso en 
los casos registrados de TBC infantil; sin embargo, en nuestra experiencia de 16 años, observamos que más de la mitad de nuestros pacientes $(65,3 \%)$ cuenta con un contacto previo con un paciente con TBC, que en su mayoría corresponde a un adulto bacilífero. Cabe destacar también que el $20 \%$ presenta al ingreso una radiografía de tórax normal, por lo que la sospecha clínica en pacientes con síntomas pulmonares y extrapulmonares junto con el antecedente de contacto se torna fundamental.

La forma de presentación de la enfermedad más frecuente fue la primoinfección, lo que concuerda con los datos obtenidos en otras experiencias clínicas nacionales y de otros países. Se ha descrito que las formas progresivas pulmonares se presentan con mayor frecuencia en pacientes de menor edad, sin embargo, en nuestros pacientes se desarrollaron con mayor frecuencia en pacientes mayores, no así las formas diseminadas, dos de las cuales se presentaron como TBC miliar, una en un recién nacido con infección probablemente connatal y la otra en un lactante con diagnóstico de inmunosupresión de etiología no descrita, siendo este último el único paciente fallecido en nuestro estudio.

También es similar a otras casuísticas la distribución de TBC extrapulmonar, siendo más frecuente la adenitis $(\mathrm{n}=8)$. El compromiso pleural que fue el segundo en frecuencia, no se describe como una de las presentaciones más importantes en otros estudios. Así mismo llama la atención la ausencia de TBC renal en nuestros pacientes lo que se puede explicar por el mayor tiempo de evolución necesario desde la primoinfección que tiene esta forma clínica (tabla de tiempo de Wallgreen).
En cuanto al tratamiento, en 45 pacientes se obtuvo un seguimiento completo, es decir, desde su inicio en la hospitalización, hasta en los controles posteriores realizados en el policlínico de broncopulmonar de nuestro hospital, confirmando en ellos su mejoría clínica y radiológica.

Creemos que en zonas de baja incidencia de tuberculosis se dificulta el diagnóstico porque no se piensa en él, y los diagnósticos diferenciales se hacen más probables.

\section{Bibliografía}

1.- NORMAS TÉCNICAS, Programa Nacional de Control de la Tuberculosis, 2005. www.minsal.cl

2.- BURGOS R, HEISE S, RÍOS R, NEUMANN I. Tuberculosis infantil en Valdivia: experiencia clínica en 16 años. Rev Chil Infectol 2002; 19: 237-44.

3.- ENARSON D A. Children and the global tuberculosis situation. Paediatr Respir Rev 2004;5 Suppl A: S1435.

4.- FIELBAUM O, HERRERA O. Enfermedades Respiratorias Infantiles. Editorial Mediterráneo. Santiago, Chile. 2002, pág. 231-42.

5.- MARAIS B J, GIE R P, SCHAAF H S, BEYERS N, DONALD P R, STARKE J R. Childhood pulmonary tuberculosis: old wisdom and new challenges. Am J Respir Crit Care Med 2006; 173: 1078-90.

6.- SINGH V. TB in developing countries: diagnosis and treatment. Paedriatr Respir Rev 2006; 7 Suppl 1: S1325.

7.- FONSECA-SANTOS J. Tuberculosis in children. Eur J Radiol 2005; 55: 202-8.

8.- WOODS C R. Advances in diagnosis and therapy of childhood tuberculosis. Paediatr Respir Rev 2006; 7 Suppl 1: S130-1.

9.- VALLEJO J, ONG L T, STARKE J R. Clinical features, diagnosis, and treatment of tuberculosis in infants. Pediatrics 1994; 94: 1-7.
Correspondencia a:

Dra. Rossana Andrea Mamani Jaches

Unidad Broncopulmonar. Hospital Roberto del Río,

Santiago, Chile

E-mail: nazorej@yahoo.com 
